\title{
DISCUSSION TO DR. WALKER'S SPEECH
}

Experiences of Reticulotomy in Epilepsy

Keiji SANo, M. D.

Dept. of Neurosurgery \& Institute of Brain Research

University of Tokyo

In the last 15 years, functions of the brain stem reticular system have been greatly elucidated by Magoun and his collaborators. The system is now widely known to modulate various kinds of neuronal activities through its ascending and descending projections. Not only the neocortex, but the limbic system comes under its influence. This report concerns the effects of lesions in the rostral brain stem reticular formation upon the pathological functional states of the brain, namcly, epileptic seizures and behavior disorders.

As for the participation of the reticular system in epileptic seizures, there are three possibilities. (1) The reticular system may act as a focus of the seizure. There have been no definite proof of this in our series. (2) The reticular system may be actively involved in conduction of seizure discharge. As Dr. Walker impressively showed in his experiments, this is also unlikely in our experience. Here is an example. In this case (Fig. 1), spiking is seen in the left rolandic 
area (the first trace). The other leads are the upper mesencephalic reticular formation (the second trace), the left putamen (the third trace) and the left lateral ventral nucleus of the thalamus (the fourth trace). By pentylene-tetrazol activation, spiking in the left rolandic area becomes more marked and rhythmic (Fig. 2), followed by discharges in the left putamen. Throughout the tonic

H. S. (1)

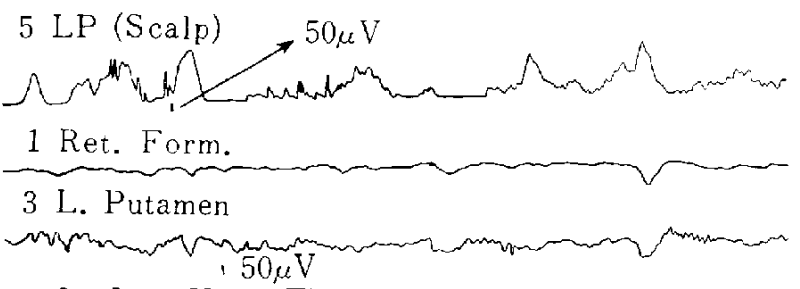

2 L. Lat. Vent. Th.
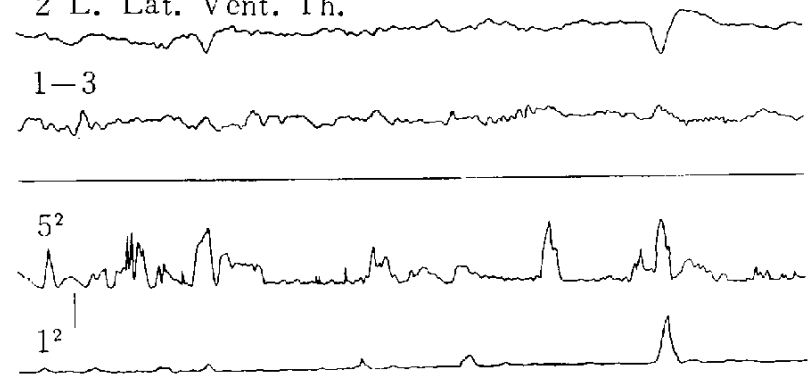

Fig. 1-5: The first trace: left rolandic area; the second trace: reticular formation; the third trace: left putamen; the fourth trace: left lateral ventral nucleus of thalamus (these are all monopolar leads); the fifth trace: reticular formation-left putamen; the seventh trace: square of potential of left rolandic area; the eighth trace: square of potential of reticular formation.

H. S. (2) 60 sec. after inj. of Ptz. (600 mg)

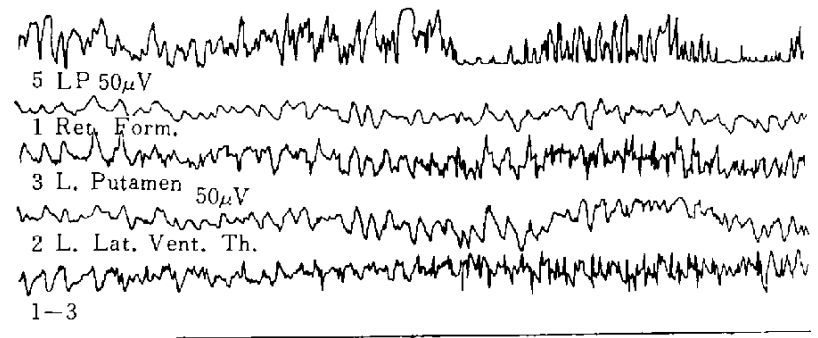

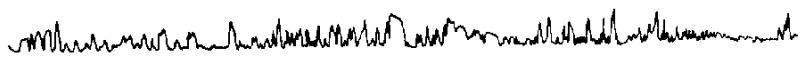
$\mathrm{L}^{2}$ $1^{2}$

Fig. 2. 
L.S. (3) 150 sec. after inj. of Ptz.

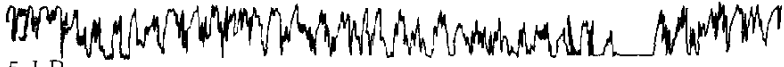 \\ 5 L.P \\ I Ret. Form.

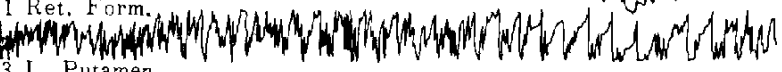

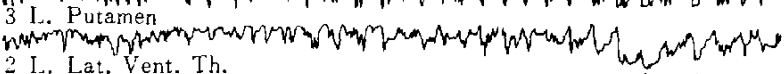

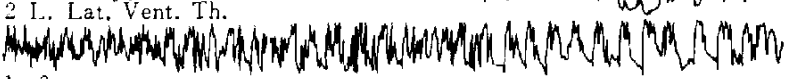 $1-3$

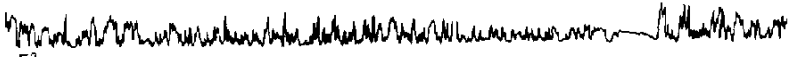

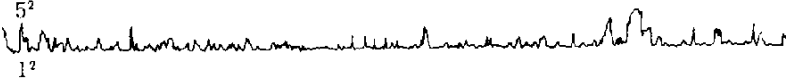

Fig. 3.

H. S. (4) 390 sec. after inj. of Ptz.

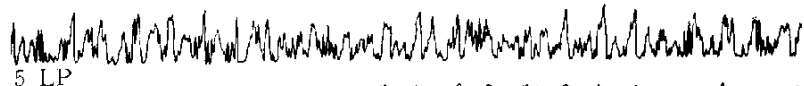

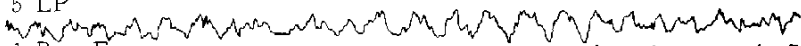

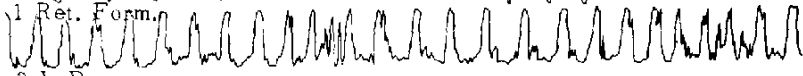

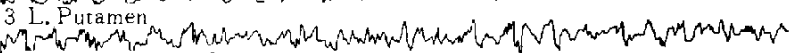

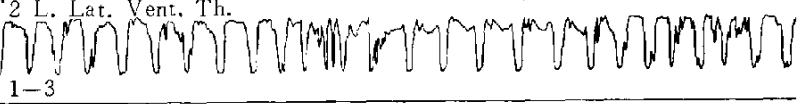

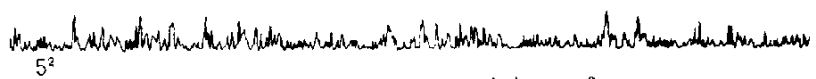

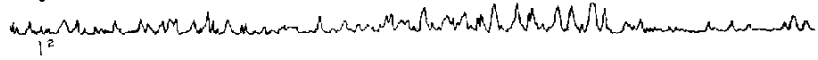

Fig. 4.

H.S. (5) 750 sec. after inj. of Ptz.

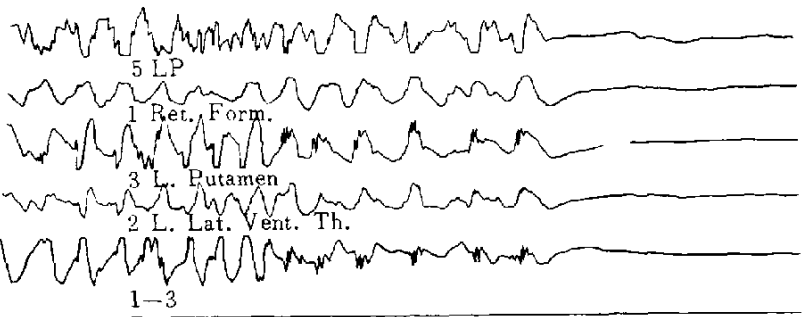

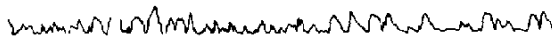

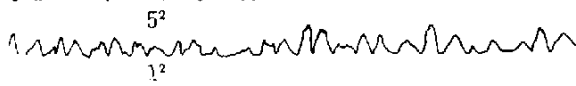

Fig. 5. 
(Fig. 3) and clonic (Fig. 4) phases of the convulsive seizure, the reticular formation remains relatively quiet except that in the clonic phase it shows slow waves of $1.5 \mathrm{c} / \mathrm{s}$, synchrnous with rhythmic sharp waves in the putamen. In the phase of extinction (Fig. 5), all leads exhibit flat traces. According to these data the reticular system does not seem to play an important role in conduction of seizure discharges. (3) The third possibility is that the reticular system may exert some influence upon firing the cortical epileptogenic focus and upon functioning of the reververating circuits. Started from this consideration, we decided to make small lesions in the upper mesencephalic reticular system in severe cases of epilepsy and behavior disorders.

So far 6 cases were submitted to the upper mesencephalic reticulotomy. The detail was reported elsewhere.") The most rostral part of the mesencephalic reticular formation around the aqucduct was electrocauterized, stereotactically, the lesion being 3 to $4 \mathrm{~mm}$ in size. The area was chosen for the site of lesion, because this area is situated rostral to the oculomotor nuclei and apart from the red nucleus. the substantia nigra, and the specific afferent and efferent systems. The lesion of the area is considered to exert bigger influences on the cerebral cortex and to yield less side effects than that of any other regions of the brain stem. The periaqueductal gray matter and the surrounding reticular formation are involved in the lesion.

Postoperatively the following three were noted: (1) tendency to calmness, (2) effects on EEG, (3) effects on seizures.

The tendency to calmness was marked in the first few weeks, gradually attenuating thereafter, but in some cases lasted for period of observation (1014 months), though in a less degree.

Most remarkable changes occurred in the patients' EEG after the operation. Generalized slow waves were noted in all cases from the first day to 3 or 4 months postoperatively. Yet the patients have been quite conscious and desynchronization of the EEG was observed when external stimuli were applied.

By the operation, ictal automatism was uninfluenced or sometimes aggravated, while convulsive seizures tended to be minor ones. In one case of convulsive seizures with spike and wave complex in the bifrontal leads, seizures and seizure discharges have completely disappeared after the reticulotomy.

The present data form the counterpart of the experiment done by our colleague, Dr. Miyasaka. ${ }^{1)}$ He stimulated the mesencephalic reticular formation and at the same time stimulated the various parts of the cerebral cortex. He concluded that the reticular formation exerted a facilitatory influence on the neocortical seizure discharges and an inhibitory influence on those of the limbic system.

If some conclusions may be drawn from these limited experiences, they will be as follows:

(1) The reticulotomy may exert favorable influences upon behavior 
problems.

(2) The effects of the reticulotomy on seizures of the limbic system are unfavorable; the seizures may sometimes increase after the operation.

(3) At the present stage, nothing definite can be stated about the effects of the reticulotomy on neocortical seizures, although there is one favorable case in the series.

\section{REFERENCES}

1) Miyasaka, M.: Electrophysiological study of seizure discharges ih neo-, paleo-, and archi-cortical systems. With special references to clinical problems of the epilepsies. Recent Advance in Research of the Nervous Systems, 5: 227-251, 1960.

2) Sano, K.: Upper mesencephalic reticulotomy in epilepsy and behavior disorders. Neurol, med.-chir., 2: 138-146, 1960. 